\title{
Methodology for preparing a cosmetic sample for the development of Microorganism Detection System (SDM) software and artificial intelligence learning to recognize specific microbial species
}

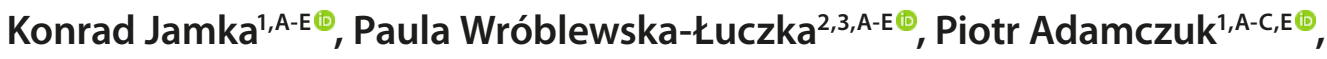 \\ Paweł Zawadzki ${ }^{4,3, A-C, E \oplus}$, Hubert Bojar ${ }^{1, A-C, E \oplus \infty}$, Grzegorz Raszewski ${ }^{1, A, C-F} \oplus$ \\ ${ }^{1}$ Institute of Rural Health, Lublin, Poland \\ 2 Department of Occupational Medicine, Medical University, Lublin, Poland \\ ${ }^{3}$ NVT Limited Liability Company, Warsaw, Poland \\ ${ }^{4}$ Institute of Theory of Electrical Engineering, Measurement and Information Systems, University of Technology, Warsaw, \\ Poland \\ A - Research concept and design, B - Collection and/or assembly of data, C - Data analysis and interpretation, \\ $D$ - Writing the article, $E$ - Critical revision of the article, F-Final approval of the article
}

\begin{abstract}
Jamka K, Wróblewska-Łuczka P, Adamczuk P, Zawadzki P, Bojar H, Raszewski G. Methodology for preparing a cosmetic sample for the development of Microorganism Detection System (SDM) software and artificial intelligence learning to recognize specific microbial species. Ann Agric Environ Med. 2021; 28(4): 681-685. doi: 10.26444/aaem/144696
\end{abstract}

\begin{abstract}
Introduction and Objective. The article presents the methodology of preparing a cosmetic sample for analysi, and the creation of a dataset for teaching artificial intelligence to recognize specific species of microorganisms in cosmetic samples in terms of compliance with the ISO standard document, to develop of the Microorganism Detection System (SDM).

Materials and method. Methodology of preparation a cosmetic sample for testing covers the steps from taking a cosmetic sample to obtaining separated living microorganisms through staining to photos, which in the final stage are used for analysis of the purity of cosmetics by SDM, as well as for learning and testing of the deep convolutional neural network (CNN) for detecting and classifying cells of specific species of bacteria, fungi and yeast in cosmetics, according to the document of standard PN-EN ISO 17516-2014:11.

Results. A new techique was devised for preparing a cosmetic sample for the development of Microorganism Detection System (SDM) software, and artificial intelligence learning to recognize specific microbial species. Based on metod demonstrated, the Intelligent algorithms of SDM proved to be effective in counting and recognizing specific microorganisms (average accuracy for Candida albicans - 97\%, Escherichia coli - 76\%, Pseudomonas aeruginosa - 70\%, Staphylococcus aureus $-85 \%)$, which are the most important species for the assessment of the purity of cosmetics. In addition, the reproducibility of the developed method was verified, and the results obtained were comparable to the breeding methods currently used, based on specific standards.

Conclusions. The experiments confirmed the high sensitivity and specificity of the SDM method, its repeatability and, above all, the comparability of the results with clasic methods of European standards.
\end{abstract}

\section{- Key words}

artificial intelligence, Microorganism Detection System, preparing cosmetic sample, analysis of the purity of cosmetics, deep convolutional neural network

\section{INTRODUCTION}

Legal regulations in the European Union obligated all producers to create cosmetics that are safe for humans to use $[1,2]$. One of the basic conditions for this safety is their microbiological purity. The causes of microbiological contamination of cosmetics may result from the lack of hygiene in the technological process, as well as the cleanliness of the raw materials used, especially quality of the water [3].

On the one hand, an excessive presence of bacterial flora in a cosmetic product reduces its quality (change of

Address for correspondence: Grzegorz Raszewski, Institute of Rural Health, Lublin, Poland

E-mail: raszewski.g@imw.lublin.pl

Received: 30.11.2021; accepted: 07.12.2021; first published: 16.12.2021 consistency, colour, unpleasant smell), while on the other hand, it may cause ailments and diseases in consumers $[4,5]$, for example, direct contact with the bacterium Pseudomonas aeruginosa present in a mascara, can cause eye damage or corneal ulceration, while Klebsiella pneumoniae from contaminated body cream can cause local dermatitis [6]. Additionally, accumulated microbial metabolites can cause local inflammatory reactions or various allergic reactions at the application sites [7].

Effective testing of the microbiological purity of cosmetics requires a number of different methods and analyzes that should meet a number of criteria [8]. In bacteriological testing of cosmetic preparations, the key factor is the time and sensitivity of the analysis, as the presence of even a small amount of the pathogen may already constitute an 
infectious dose. The most important limitations regarding the usefulness of microbiological testing is to quickly identify the bacterial contaminations and treat them before they do irreversible damage [7].

Traditional microbiological tests and methods used for the qualitative and quantitative detection of pathogens in cosmetic preparations are sensitive, inexpensive and provide fully reliable information that meets the requirements imposed on cosmetics producers by the Regulation of the European Parliament and of the Council of Europe (No. $1223 / 2009$ / EC of 30.11.2009) and national standards (including PN-EN ISO 17516: 2014-11) [1, 2].

However, their disadvantage is the high labour-intensity and time-consuming process associated mainly with a long 24-72 hours of incubation, and growth and multiplication of the microorganism [9]. They often require additional culture on differentiation media, preparation of specimes and their microscopic verification, and/or the analysis using other diagnostic techniques, such as flow cytometry, molecular PCR methods, or technologies using the so-called biosensors [10, 11]. Additionally, to perform such analyzes, a well-equipped microbiological laboratory and highly qualified technical staff are required. These factors make the monitoring and assessment of the microbiological condition of the cosmetics in the production process, in accordance with applicable standards and regulations, a specialist process, increasing the cost of cosmetic production and therefore often unavailable to small production companies $[9,12]$.

Despite the continuous development of research, creating detection systems that can specifically distinguish target bacteria in a matrix with multiple organisms, with characteristics of adaptability and sensitivity to detect different microorganisms, while working online in real time, is a big challenge [13-16]. The answer to this problem may be the Microorganism Detection System (SDM) for the detection of specific microorganisms in accordance with the PN-EN ISO 17516-2014: 11 standard [1], developed by specialists from the consortium of the NVT Sp. z o.o. and the Institute of Rural Health in Lublin, Poland. This system in an innovative way in real time which determines the acceptable levels of bacterial contamination of cosmetic products, through the use of artificial intelligence (AI) to optimize the microbiological control process of cosmetics with the use of algorithms for microscopic image interpretation. [17]. In addition, the SDM can remotely perform microbiological analysis, which is an international breakthrough in the way that routine microbiological safety testing of industrial products is performed [17].

An integral part of the presented SDM, in addition to its SDM programme part, is the methodology of preparing a cosmetic sample, developed by the research team from the Institute of Rural Health. Also, the methodology covers the creation of a dataset consisting of photos of Candida albicans, Escherichia coli, Pseudomonas aeruginosa and Staphylococcus aureus, separately and in various bacterial matrix from microscope slides. The dataset in the final stage were used to design SDM software and teach the deep convolutional neural network (CNN) for detecting and classifying cells of specific species of bacteria, fungi and yeast in cosmetics $[15,16,17]$.

The aim of this article is to propose the methodology of preparing a cosmetic sample for the development of SDM software and artificial intelligence learning to recognize specific microbial species according to the document of standard PN-EN ISO 17516-2014:11 [1].

\section{MATERIALS AND METHOD}

The methodology of preparation of a cosmetic sample for testing covers the steps from taking a cosmetic sample to obtaining separated living microorganisms through staining to photos, which in the final stage are used for analysis of target microorganisms in samples of cosmetics by the artificial intelligence of the SDM [17]. The SDM project assumed the development and adaptation of such a method of preparing a cosmetic sample as to omit the stage of microbial cultivation, which would speed up assessment of the microbiological purity of cosmetics.

Methods for obtaining separated live microorganisms and its staining from cosmetics. The research team from the Institute of Rural Health tested numerous variations of the methods, based primarily on the methods of concentration: flotation, sedimentation, centrifugation in a concentration gradient, and filtration to obtaining separated living microorganisms.

Next, a method was sought of complex staining, so that in one microscopic preparation it was possible to simultaneously evaluate bacteria, yeasts and moulds, and the photos taken were useful for creating the SDM $[16,17]$. Many simple dyeing methods were tested, including: Congo red, nigrosine, safranin, basic fuchsin, malachite green, crystal violet, eosin, and Sudan black. How each dye behaved was assessed in staining bacteria, yeasts and moulds, and which dyes would be useful for detecting microorganisms through artificial intelligence. Finally, after numerous experiments with the use of various methods of staining, fixing the preparation or the use of various types of filters, the final method of staining was approved. This method consists in staining filter purified microorganisms from a cosmetic sample on a membrane, performed using a vacuum filtration kit and a modified Gram method. The obtained filter with stained microorganisms is dried and transferred to a microscope slide for photos to be taken which are analyzed for specific microbial species using the SDM.

Creation of a deep-learning dataset. To create the dataset, data consisting of 5 sets of images were used. Each set contained 5 images taken of 19 microorganisms (within 4 specific microorganisms): Bacillus subtilis, Candida albicans, Candida dubliniensis, Candida glabrata, Candida tropicalis, Escherichia coli, Enterococcus faecalis, Klebsiella pneumoniae, Listeria monocytogenes, Morganella morganii, Pseudomonas aeruginosa, Proteus mirabilis, Proteus vulgaris, Rhodococcus equi, Streptococcus agalactiae, Staphylococcus aureus, Staphylococcus epidermidis, Serratia marcescens and Salmonella typhimurium from ATTC and NTCT collections. Spore photos of various molds were also used to enrich the base $[17,18]$.

This dataset was used to train and test the artificial intelligence (AI) algorithms of SDM for recognizing and counting specific microorganisms: Candida albicans, Escherichia coli, Pseudomonas aeruginosa and Staphylococcus aureus, which are the most important species for assessment of the purity of cosmetics, as well as assigning all 
microorganisms into the following groups: bacteria, yeasts and moulds [17].

The suspensions of microorganisms in Ringer's fluid or PBS buffer (Merck KGaA, Darmstadt, Germany) with known cell numbers were used to prepare the original photos, which were then stained with a modified Gram's method (as in the cosmetic's sample). The concentration of the suspension was measured densitometrically, and the suspensions of microorganisms diluted in Ringer's fluid or PBS to a final concentration of 1/100 CFU per $0.1 \mathrm{~mL}$. From $0.1 \mathrm{~mL}$ of diluted suspensions, after Gram staining the original photos were prepared, as well as counting cells in the suspensions they were spread on a Tryptone-Soja agar or SabourandDextrose plate (Biomaxima, Lublin, Poland), and incubated at the appropriate temperature.

Microscopic photos of microorganisms. Numerous photos from microscopic slides stained with Gram staining modifications were taken. Next, photos from microscopic slides were sent to the Consortium member NVT for the purpose of creating SDM software and learning artificial intelligence to recognize specific species of microorganisms.

The images were taken with an Olympus CX43 Biological Microscope equipped with a SC180 camera (Olympus Corporation, Japan). They were evaluated using a 100 times objective under oil-immersion (Olympus RI $n=1.516$, Japan). The images were taken at the maximum resolution: 4912x3684, 96 dpi.

\section{RESULTS AND DISCUSSION}

The research team of the Institute of Rural demonstrated the methodology of preparing a cosmetic sample for rapid and in real-time detection by SDM of specific microorganisms accordance with the PN-EN ISO 17516: 2014-11standard, (Candida albicans, Escherichia coli, Pseudomonas aeruginosa, Staphylococcus aureus) and giving an answer whether the tested product complies with it. This standard defines the acceptable levels of microbiological contamination in accordance with the category of the cosmetic [1].

The final, validated by us methodology for the preparation of cosmetic samples consists of the following steps: weighing $1 \mathrm{~g} / 1 \mathrm{ml}$ of the sample, adding Tween 80 and Ringer's fluid (or PBS buffer), vortexing to obtain a homogeneous suspension, applying a sucrose density gradient to a properly prepared gradient, placing it in water bath, centrifugation, gradient solidification, removal of the upper phase, addition of sterile distilled water, centrifugation, placing the sediment in the Percoll density gradient, centrifugation, purified microorganisms are at the phase boundary of individual densities from where $1 \mathrm{ml}$ is taken for the next stage - staining on the filter.

Staining on the filter (membrane filter Sartorius, 0.4 $\mu \mathrm{m}$, Germany) was performed using a vacuum filtration kit (Diaphragm pump LABOPORT ${ }^{\circledast}$ N86 KT.18, KNF, Germany). The sample is passed through a filter, fixed with ethyl alcohol, stained with crystal violet, rinsed with distilled water, Lugol's solution is added, decolorized with ethyl alcohol, rinsed with water, stained with carbol fuchsin, rinsed with distilled water. Each of the mentioned staining / washing steps is carried out for an appropriate time with an appropriately set vacuum pump, and the added reagent is in a specific, experimentally verified volume. The thus obtained filter with stained microorganisms is dried and transferred to a microscope slide, takes microscope photos. The photos we made, were used for creating and learning of algorithms of SDM for microscopic image interpretation [19-21].

It is worth noting that, the exact methodology of sample preparation and staining together with the software of the SDM form mutually dependent parts and are provided only to cosmetic companies cooperating with the Consortium.

Based on showed method of separation, concentration and staining on the filter, the Intelligent algorithms of SDM turns out to be effective in counting and recognizing specific microorganisms: Escherichia coli, Staphylococcus aureus, Candida albicans, Pseudomonas aeruginosa, which are the most important species for the assessment of the purity of cosmetics and in assigning all analyzed microorganisms to the following groups: bacteria, yeast and mold.

The results of CNN, for the test data, obtained an average accuracy of $80 \%$, but a more detailed analysis showed that for some microorganisms the obtained results were much higher (Candida cells over 95\% accuracy) or lower (Proteus vulgaris cells 59\% accuracy). However, from the point of view of the SDM, the most important point was to recognize specific microorganisms: Candida albicans, Escherichia coli, Pseudomonas aeruginosa, and Staphylococcus aureus. For these bacterial cells, an overall identification accuracy for Candida albicans - 97\%, Escherichia coli - 76\%, Pseudomonas aeruginosa - 70\%, Staphylococcus aureus $85 \%$, were obtained [17]. In addition, the reproducibility of the developed method was verified and the results obtained were comparable to the breeding methods currently used, based on specific standards. In summary, the SDM system proved to be effective in recognizing and counting the microorganisms listed in the document of standard PN-EN ISO 17516-2014:11 [1, 17].

As an option for testing the methods useful for bacterial separation and concentration, chemical, physical, physicochemical or biological approaches were used. For this purpose, flotation, sedimentation, centrifugation in a concentration gradient, filtration on filters, and some other techniques with numerous modifications, were used [21, 22, $23,24]$. The separation and concentration schemes, applied singly or in combination, were aimed to provide a sample with a high recovery of viable target microorganisms, and its cleaning from various pollutants [21].

After trying different techniques for staining microorganisms, it was decided to use the Gram method with numerous modifications [20,25, 26, 27]. Gram staining is the most basic technique for bacterial identification, as well as a method for differentiating the bacterial species based on their chemical and physical properties into Gram-positive and Gram-negative groups. However, not all microorganisms can be definitively classified by this technique, and the staining method is a valuable diagnostic tool for determining the identity of a specific microbiological sample. The results of the $\mathrm{CNN}$ for the test data confirm the effectiveness of this staining technique. The accuracy rate for the classification of the photos was from 70\% (Pseudomonas aeruginosa) to $97 \%$ (Candida albicans), which shows that the parameters of staining assessed by the algorithm can differentiate between microorganisms groups. The differences in accuracy probably occurred due to limitations in the original training data. 
The procedure from the Gram staining of cells to automated image analysis were used have been used in some studies. Experimental research using the CNN algorithm in image processing to analyze bacterial cells using Gram's differential staining is very limited. By using deep learning, the computer system was taught to recognize and distinguish 33 species of bacteria Gram stained with an accuracy of recognition of $97.24 \pm 1.07 \%$ [28]. This procedure for staining was also proposed in 2018 by Smith et al. [20] in a study using CNN for the automated analyses of cells from blood culture. The authors used deep neural networks and the Gram staining method, by which they obtained sensitivity and specificity of $98.4 \%$ and $75.0 \%$, respectively, for Gram-positive cocci in chains and pairs, $93.2 \%$ and $97.2 \%$ for Gram-positive cocci in clusters, and $96.3 \%$ and $98.1 \%$ for Gram-negative rods. They also indicated that the analysis of the image of Gramstained microbes by artificial intelligence may have a unique application in clinical microbiology [20].

\section{CONCLUSIONS}

This article proposed a methodology for preparing a cosmetic sample for the quick detection and classification of specific microbial species by SDM, according to the document of standard PN-EN ISO 17516-2014:11 [1], with the use of deep neural networks. The SDM system proved to be effective in recognizing and counting Candida albicans, Escherichia coli, Pseudomonas aeruginosa, and Staphylococcus aureus, which are the most important species for the assessment of the purity of cosmetics, and in assigning all microorganisms to the following groups: bacteria, yeasts and mould.

New methods of testing the microbiological purity of various products, including cosmetics, are constantly being sought. Ensuring the purity of products is important for producers as well as consumers who are increasingly aware of the choice of products. An important issue is also the reduction of the analysis time, which is important for the manufacturer who wants quick confirmation of the purity of the obtained substrate, or the purity of the finished product. Deep machine learning and the analysis of microscopic images seem to be promising tools in microbiological analysis, which is confirmed by the presented research, as well as the results of other authors [20,28-31]. Machine learning can significantly shorten the analysis time and reduce its costs while ensuring a high repeatability of results.

\section{Acknowledgements}

The research was co-financed by the European Union from the European Regional Development Fund under the Intelligent Development programme, implemented under the competition 1 / 4.1.4 / 2018 / POIR: 'Microorganism detection system - SDM' - National Centre for Research and Development.

\section{REFERENCES}

1. Norma PN-EN ISO 17516:2014-11: Kosmetyki-Mikrobiologia-Limity mikrobiologiczne.

2. Regulation (EC) No 1223/2009 of the European Parliament and of the Council of 30 November 2009 on cosmetic products. https://eur-lex. europa.eu/eli/reg/2009/1223/oj (access: 2021.11.07).
3. Halla N, Fernandes IP, Heleno SA, Costa P, Boucherit-Otmani Z, Boucherit $\mathrm{K}$, et al. Cosmetics preservation: a review on present strategies. Molecules. 2018; 23(7): 1571. doi: 10.3390/molecules23071571

4. Campana R, Scesa C, Patrone V, Vittoria E, Baffone W. Microbiological study of cosmetic products during their use by consumers: health risk and efficacy of preservative systems. Lett Appl Microbiol. 2006; 43(3): 301-6. doi: 10.1111/j.1472-765X.2006.01952.x

5. Bilal M, Mehmood S, Iqbal Hafiz MN. The Beast of Beauty: Environmental and Health Concerns of Toxic Components in Cosmetics. Cosmetics 2020; 7: 13. doi: 10.3390/cosmetics7010013

6. Findley K, Grice EA. The skin microbiome: a focus on pathogens and their association with skin disease. PLoS Pathog. 2014; 10(10): e1004436-e. doi: 10.1371/journal.ppat.1004436

7. Bashir A, Lambert P. Microbiological study of used cosmetic products: highlighting possible impact on consumer health. J Appl Microbiol. 2020; 128(2): 598-605. doi: 10.1111/jam.14479

8. Skowron K, Jakubicz A, Budzyńska A, Kaczmarek A, Grudlewska K, Reśliński A, Gospodarek-Komkowska E. Microbiological purity assessment of cosmetics used by one and several persons and cosmetics after their expiry date. Rocz Panstw Zakl Hig. 2017; 68(2): 191-197.

9. Russell AD. Challenge testing: principles and practice. Int J Cosmet Sci. 2003; 25(3): 147-53. doi: 10.1046/j.1467-2494.2003.00179.x

10. Nemati M, Hamidi A, Maleki Dizaj S, Javaherzadeh V, Lotfipour F. An Overview on Novel Microbial Determination Methods in Pharmaceutical and Food Quality Control. Adv Pharm Bull. 2016; 6(3): 301-308. doi: 10.15171/apb.2016.042

11. Huang F, Zhang Y, Lin J, Liu Y. Biosensors Coupled with Signal Amplification Technology for the Detection of Pathogenic Bacteria: A Review. Biosensors (Basel). 2021 Jun 9; 11(6): 190. doi: 10.3390/ bios 11060190

12. Michalek IM, John SM, Caetano dos Santos FL. Microbiological contamination of cosmetic products-observations from Europe, 2005-2018. J Eur Acad Dermatol Venereol. 2019; 33: 2151-2157.

13. The Scientific Committee on Consumers Safety, Directorate-General for Health and Consumer Protection of the European Commission. The SSCS's Notes of Guidance for the Testing of Cosmetic Ingredients and Their Safety Evaluation, 10th ed. Brussels, Belgium: European Comission; 2018.

14. Szeląg B, Drewnowski J, Łagód G, Majerek D, Dacewicz E, Fatone F. Soft Sensor Application in Identification of the Activated Sludge Bulking Considering the Technological and Economical Aspects of Smart Systems Functioning. Sensors 2020, 20, 1941; doi: 10.3390/s20071941

15. Yuanyi Z, Wang J, Peng J, Zhang L. Anchor box optimization for object detection. In The IEEE Winter Conference on Applications of Computer Vision, 2020. p. 1286-1294.

16. Alzubaidi L, Zhang J, Amjad I, Humaidi J, Al-Dujaili A, Duan Y, et al. Review of deep learning: concepts, CNN architectures, challenges, applications, future directions. J Big Data. 2021; 8(1): 53. doi: 10.1186/ s40537-021-00444-8

17. Zawadzki P, Adamczuk P, Jamka K, Wróblewska-Łuczka P, Bojar H, Raszewski G. The Microorganism Detection System (SDM) for microbiological control of cosmetic products. Ann Agric Environ Med. doi: $10.26444 /$ aaem/144668

18. Strains specified by official microbial assays, American Type Culture Collection [ATCC]. https://www.atcc.org/ /media/ PDFs/Marketing\%20Material/Microbiology/ Pharmaceutical\% 20Microbiology.ashx, 2017. (access: 2021.11.07).

19. Smith KP, Kirby JE Image analysis and artificial intelligence in infectious disease diagnostics. Clin Microbiol Infect. 2020 Oct; 26(10): 1318-1323. doi: 10.1016/j.cmi.2020.03.012

20. Satyanarayana KV, Rao NT, Bhattacharyya D, Hu YC. Identifying the presence of bacteria on digital images by using asymmetric distribution with k-means clustering algorithm. Multidimens Syst Signal Process. 2021 Oct 9: 1-26. doi: 10.1007/s11045-021-00800-0

21. Smith KP, Kang AD, Kirby JE. Automated interpretation of blood culture Gram stains by use of a deep convolutional neural network. J Clin Microbiol. 2018; 56 (e01521): 17.

22. Stevens KA, Jaykus LA. Bacterial separation and concentration from complex sample matrices: a review. Crit Rev Microbiol. 2004; 30(1): 7-24. doi: 10.1080/10408410490266410

23. Fukushima H, Katsube K, Hata Y, Kishi R, Fujiwara S. Rapid separation and concentration of food-borne pathogens in food samples prior to quantification by viable-cell counting and real-time PCR. Appl Environ Microbiol. 2007; 73(1): 92-100. doi: 10.1128/AEM.01772-06

24. Pertoft H. Fractionation of cells and subcellular particles with Percoll. J. Biochem. Biophys. Methods. 2000; 44: 1-30. https://doi.org/10.1016/ S0165-022X(00)00066-X 
25. Garbacz M, Malec A, Duda-Saternus S, Suchorab Z, Guz Ł, Łagód G Methods for Early Detection of Microbiological Infestation of Buildings Based on Gas Sensor Technologies. Chemosensors. 2020; 8: 7. doi $10.3390 /$ chemosensors 8010007

26. Romero S, Schell RF, Pennell DR. Rapid method for the differentiation of gram-positive and gram-negative bacteria on membrane filters. J Clin Microbiol. 1988 Jul; 26(7): 1378-82. doi: 10.1128/jcm.26.7.13781382.1988

27. Saida H, Ytow N, Seki H. Photometric Application of the Gram Stain Method To Characterize Natural Bacterial Populations in Aquatic Environments. Appl Environ Microbiol. 1998; 64(2): 742-7. doi: 10.1128/ AEM.64.2.742-747.1998

28. Wang H, Kodmir HC, Qiu Y, et al. Early detection and classification of live bacteria using time-lapse coherent imaging and deep learning. Light Science Applications. 2020; 9(118): 1-17.
29. Zieliński B, Plichta A, Misztal K, Spurek P, Brzychczy-Włoch M, Ochońska D. Deep learning approach to bacterial colony classification. PLoS ONE. 2017; 12(9): e0184554. doi: 10.1371/journal.pone.0184554 30. Maruthamuthu MK, Raffiee AH, De Oliveira DM, Ardekani AM, Verma MS. Raman spectra-based deep learning: A tool to identify microbial contamination. Microbiologyopen. 2020; 9(11): e1122. doi: $10.1002 / \mathrm{mbo} .1122$

31. Rani P, Kotwal S, Manhas J, Sharma V, Sharma S. Machine Learning and Deep Learning Based Computational Approaches in Automatic Microorganisms Image Recognition: Methodologies, Challenges, and Developments. Arch Comput Methods Eng. 2021; 1-37. doi: 10.1007/ s11831-021-09639-x

32. Zhang Y, Jiang H, Ye T, Juhas M. Deep Learning for Imaging and Detection of Microorganisms. Trends Microbiol. 2021; 29(7): 569-572. doi: 10.1016/j.tim.2021.01.006 